\title{
Design and Development of Speed Controller for Drowsy Drivers
}

\author{
Mrs.N.Neelima ${ }^{1}$,Ms.S.Sri Lakshmi ${ }^{2}$, Mr. T.Jaya Vardhan ${ }^{3}$ \\ ${ }^{1}$ H.O.D, Dept of ECE,Malla Reddy Institute of Technology \& Science,Secunderabad,A.P,India \\ ${ }^{2}$ Asst Prof, Dept of ECE,Malla Reddy Institute of Technology \& Science,Secunderabad,A.P,India \\ ${ }^{3}$ M.Tech, Dept of ECE,Malla Reddy Institute of Technology \& Science,Secunderabad,A.P,India
}

\begin{abstract}
A Real-Time accident prevention system has been proposed in which the drowsy condition of the driver can be detected and Speed Controlling will be performed for each condition automatically. There are different ways to detect drowsiness one of them are using camera that points directly towards the driver's face and monitors the driver's eyes in order to detect fatigue. We have developed a drowsy driver detection system using Brain Computer Interface, the system deals with EEG Signal obtained from the brain, when rhythms are plotted on PC we can see the fluctuations of rhythms when subject is falling to drowsy or deep sleep in accordance with a appropriate voltage under normal condition and drowsy condition are read on software application, using these voltage under two states we have developed a alert system and speed controlling for drowsy driver. However, the current BCI system is developed to detect the drowsiness, cognitive state and when drowsy state occurs a warning tone is employed to alert him from the drowsy state, in some cases when driver don't respond to the warning tone, the speed of vehicle rapidly reduced and finally the vehicle will be stopped.
\end{abstract}

Keywords : Brain Computer Interface, Cognitive,Drowsy,EEG,Speed Controlling.

\section{INTRODUCTION}

The Technology made everything shorten, in concern in the field of transportation the automobiles has tremendously grown up every year \& also the road accident increased a lot due to drowsy driving, Although advance technology in transportation researchers ensuring safety, however the safety of a vehicle is an important task for automotive industries \& researchers .Alert systems for preventing accidents is one of the design of safety systems, these alert systems for preventing accidents is an attracting in the public [ 2 ]. In concern safety is first priority for the public, several people are dead and some are seriously injured due to drowsiness, $55 \%$ related to drowsiness causing serious accidents on the roads, falling to drowsy drivers lose their ability of controlling vehicles. Same situations where a driver doesn't respond to alert systems, in that case the driver encounter to accidents, therefore it is essential to develop a speed controlling when driver falling into drowsy for drowsy related accident prevention. There are many methods which have been developed alert systems and being used for drowsiness detection by means of physiological parameters like pulse rate, eyelid movement and head movement, perhaps current technologies have developed and implemented eye blinking, eye closure and head movement for monitoring alertness. The main aim of the project is to develop a prototype of speed controlling for drowsy drivers, the focusing point in the design is monitoring drowsier in the form of voltage levels of EEG signals of both under normal condition and drowsy condition, considering two different voltage levels can be taken as reference \& speed controlling mechanism is developed which is enough to avoid a car accident in drowsy condition.

\section{RELATED WORK}

Several Techniques developed to analyze the driver drowsiness [9]-[11]. In this paper the driver drowsiness \& warning tone is based on the monitoring changes in the human cognitive state \& provide biofeedback to the driver, when drowsy state occurs [14]-[16] . In this study a real time drowsy detection algorithm is developed and most previous case for EEG based drowsy detection is designed and developed, a detection and alert model for all subjects. Although these models may not be able to predict accurately in the cognitive state. The subject dependent system shows variations in EEG Spectra, due to different factors such as electrode displacement, skin electrode impedance and external noises, the EEG spectra in theta rhythm reflects the changes in the cognitive state these changes motivated us to develop warning system and speed controlling based on EEG Spectra in theta rhythm with variations in the voltage levels for different EEG spectra. The proposed system comprises of 3 stages, the first stage is EEG data processing which includes capturing raw EEG voltages under normal \& drowsy condition and data processing over the controller. The second stage is capable of judging drowsiness, 7.5 Micro Volts are considered as the normal condition i.e. wake up state \& above 10 Micro Volts is considered as drowsy condition, during the experiment we monitored the variations in brain rhythms in both cases i.e normal \& drowsy condition. In the final stage we programed the controller in such a way when 7.5 Micro Volts as an interrupt when it arises it is read as normal \& displayed on LED and 
when it is in drowsy condition i.e 10 Micro Volts set as drowsy when above 10 Micro Volts subject falling to sleep \& buzzer triggered to alarm and driver is alerted.

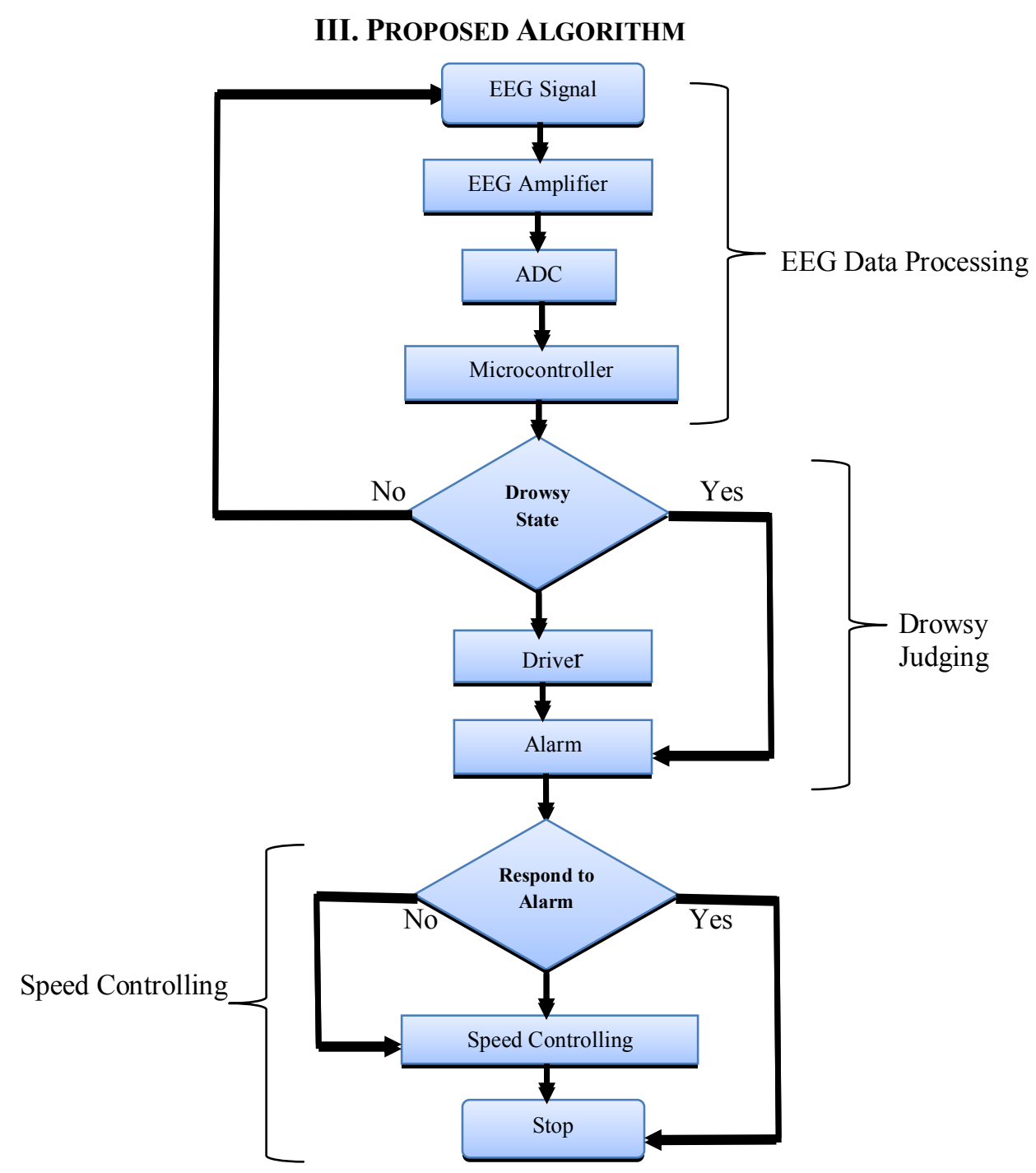

Figure 1. Proposed Algorithm

In the above figure 1 , the first stage is EEG data processing which is done by using 3 electrodes in that 2 nd electrodes placed on frontal lobe \& 3rd electrode to ground, these three electrodes connected to EEG Amplifier and the output is given to PC and noted down the voltage for each rhythm. The second stage is the drowsiness, the appropriate amplitude levels are read on the software application, this window is scaled to detect the behavior of brain rhythms under different condition. The Final stage is alert system \& speed controlling, Similarly the voltage levels are captured from the EEG Software Application. To detect the drowsy condition we first detect the normal state and cognitive state. We have connected the electrode to subject head, start recording the brain rhythms. The next step is to capture the voltage levels in accordance with fluctuations in brain rhythms, monitoring both values after 7.5 Micro Volt driver falling to drowsy state slowly, when driver is completely under drowsy state. i.e 10 Micro Volts are recorded and above this state 10 Micro Volts are sleepy state and alarm is triggered to alert the driver from drowsy. In case if the driver not responding to alert tone the speed controlling mechanism will control the speed of the vehicle, example $60 \mathrm{Kmph}$ to $20 \mathrm{Kmph}$. We have set the voltages as :

$$
\begin{gathered}
\text { V1 }=7.5 \text { Micro volts and } \\
\text { V2 }=10 \text { Micro Volts, therefore }
\end{gathered}
$$

Drowsy Falling State $=\mathrm{V} 2-\mathrm{V} 1=10-7.5$ Micro Volts

Drowsy Falling State $=2.5$ Micro Volts

The Above V2 is Completely Sleep State and V2-V1 Drowsy Falling State. 


\section{1.experiment design}

\section{RESULTS \& DISCUSSIONS}

To verify the Feasibility of our propose speed controlling for drowsy driver, an experiment is designed for testing here a car is suspended on strong base so that the car can take a motion. The controller we used is AT89C52 which is a low-power, high-performance CMOS 8-bit microcomputer with 8K bytes of Flash programmable and erasable read only memory (PEROM). The device is manufactured using Atmel's highdensity nonvolatile memory technology and is compatible with the industry-standard 80C51 and 80C52 instruction set and pin out.

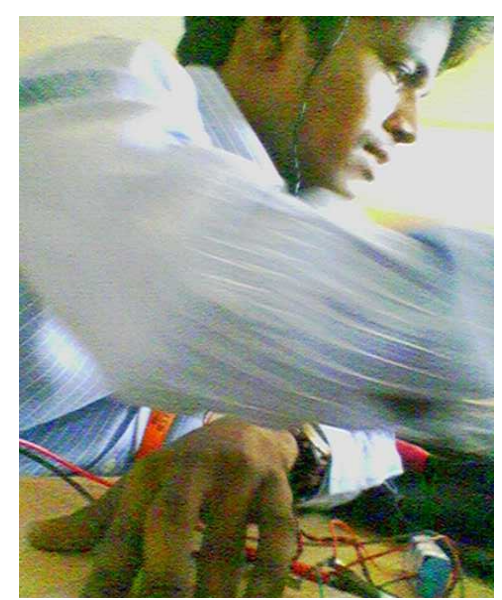

Figure 2. Experimental Setup

The AVR core combines a rich instruction set with 32 general purpose working registers. All the 32 registers are directly connected to the Arithmetic Logic Unit (ALU), allowing two independent registers to be accessed in one single instruction executed in one clock cycle. The resulting architecture is more code efficient while achieving throughputs up to ten times faster than conventional.

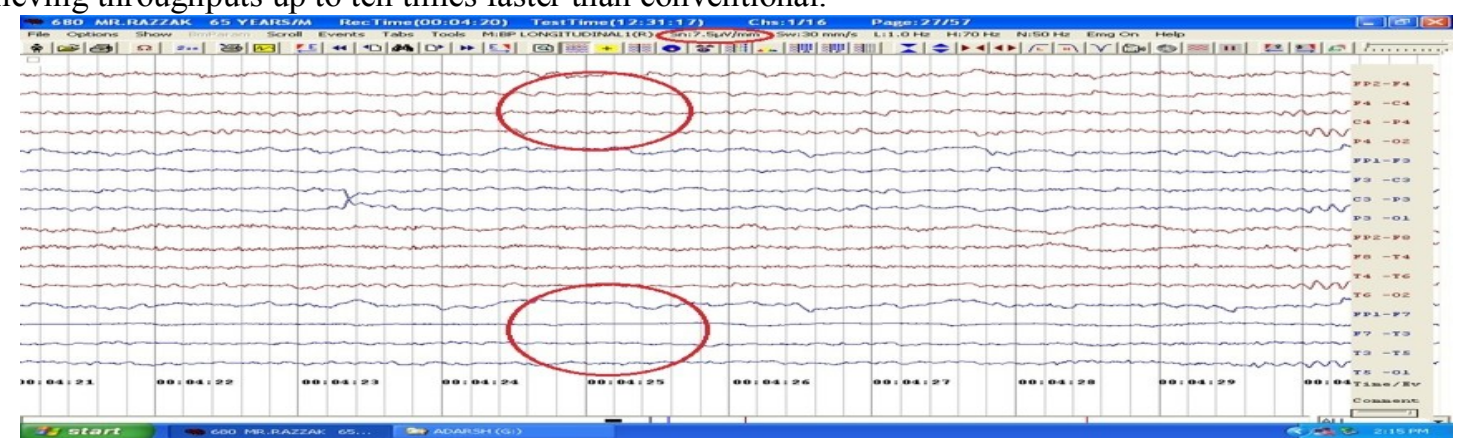

Figure 3. Brain Rhythms Under Normal Condition

the electrodes used is about 23 channel electrode, the application enabled with frequency reading, voltage reading, and recording the rhythm, Rhythm are grouped by frequency amplitudes are about $100 \mu \mathrm{V}$ max, in general the above picture rhythm's captured in normal position, the subject is $65 \mathrm{yr}$ 's old, the picture depicts that under normal condition the rhythms are reading normal here by we can see rhythm's under normal condition showing as 7.5 micro volts .

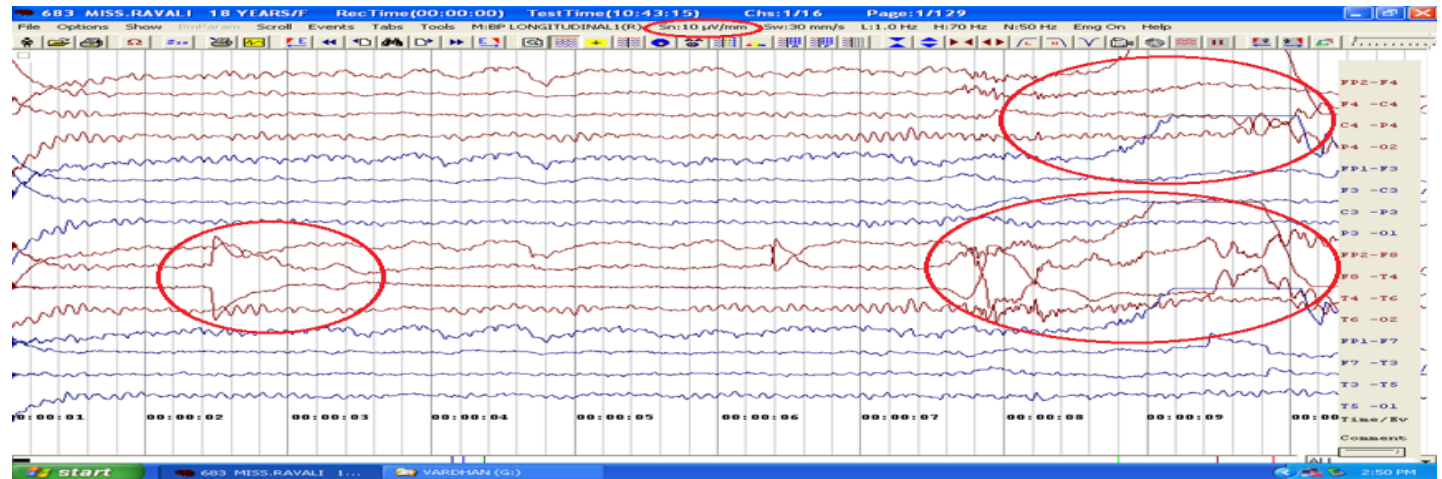

Figure 4. Brain Rhythms Under Drowsy Condition 
The above picture rhythm's captured in drowsy position, the subject is $18 \mathrm{yr}$ 's old , the picture replicates that under drowsy condition and rhythm's are reading normal condition here by we can see rhythm's under Drowsy condition it is showing as 10 micro volts .

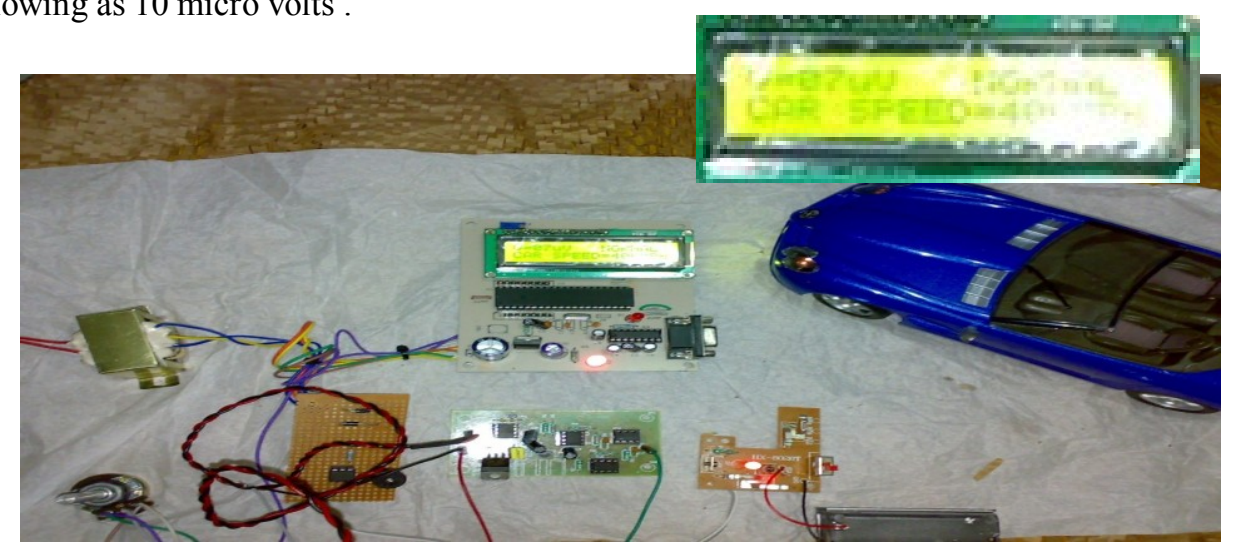

Figure 5. Speed Control Under Normal Condition

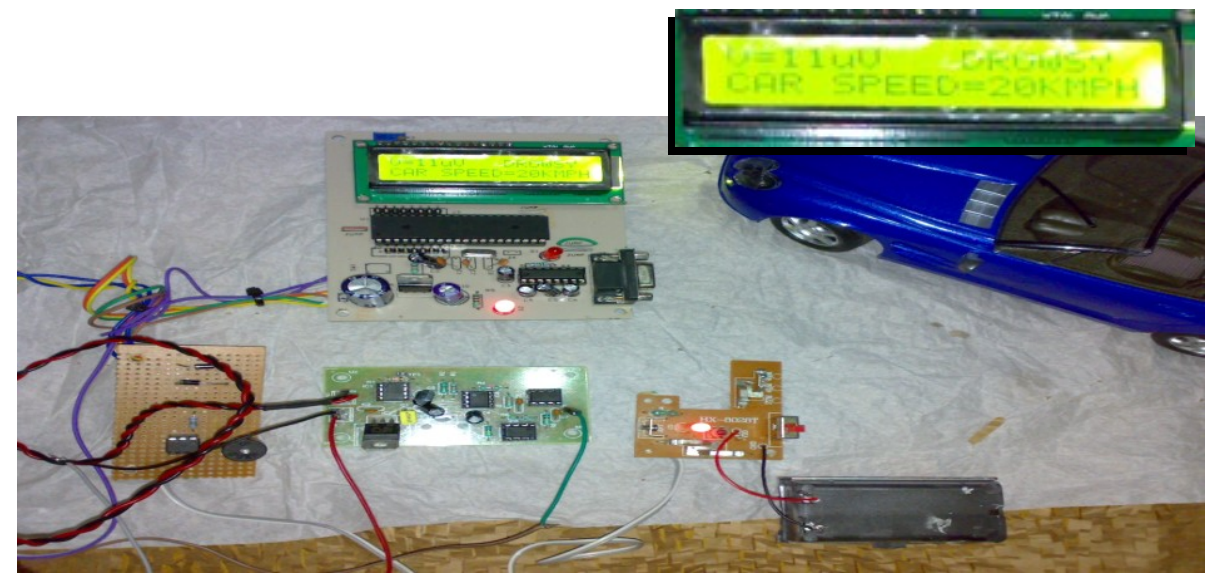

Figure 6. Speed Control Under Drowsy Condition

The above Fig $5 \& 6$ depicts the experimental setup of speed controlling under normal and Drowsy condition. The Data transmission from Car and the module is carried out with RF Transceiver with $42 \mathrm{MHz}$ High Frequency . The acceleration of the car controlled by using optoisolator, when the accelerator is pushed down i.e speed increases in the same time, the two voltages fluctuate from high and low (under drowsy and normal condition ) are connected to optoisolator, during normal condition the behavior of the optoisolator isolate to a $\operatorname{logic} 1$ state and during drowsy condition optoisolator isolate to logic 0 , the speed of car toggle between these states and more delay is applied for further reduction in the speed of car (i.e $20 \mathrm{kmph}$ ) comes down under drowsy condition.

\section{CONCLUSION}

The Noninvasive System is to localize the brain rhythms and monitor the drowsy\& the speed controlling of car is developed. During monitoring the system is able to judge the variations in rhythms in accordance with the voltage under normal \& drowsy condition. When a driver is drowsy above the voltage variations is up to 10 Micro Volts in theta rhythm, an alert signal is triggered \& speed of the vehicle rapidly decreased. This technology is completely accident prevent system \& highly secured this can be used for transportation involving in shipping the goods from different locations, as it is wired communication from head to external device, at some conditions driver may feel disgust in continuously wearing the electrodes, the motto of this paper is to prevent accidents from drowsy .

\section{Acknowledgement}

Thanks to Lord Sai Baba for blessing us with the strength and patience to complete this project. We wouldlike to thanks Dr. K.Ravindra, Principal of MRITS for Extreme Support in encouraging Students, Dr.Jayaprada,SC-D,ANURAG,DRDO for her support,my Sincere thanks to Dr.Ramdas,Superintendent OGH,Hyd, Dr. Suma (Dept of Neurology) and Mr. Anil (EEG Lab Technician) for their Extraordinary Support and advice throughout the project. Thanks to Mr.Nagaravi and my very special thanks to Mr.Srinivasa Rao Bhadharla for providing EEG related equipment's and special thanks to lab faculty and faculty of MRITS . 


\section{References}

[1] Brown ID. "Driver fatigue and road safety. Alcohol, Drugs and Driving" 1993.

[2] J.D Wu, T.R Chen "Development of a drowsiness warning system based on the fuzzy logic images analysis" Expert Systems with Applications 34 (2008), pp. 1556-1561.

[3] Brown ID "Driver fatigue Human Factors", 1994. pp:298-314.

[4] G.Maycock. "Sleepiness And Driving : Experience of UK Cars Drivers" vol 51996.

[5] L.A.Rayner ,J.A.Horne. 23 June 1997."Falling asleep whilst driving drivers aware of prior sleepiness.

[6] S. Makeig and M. Inlow, "Lapses in alertness: Coherence of fluctuations in performance and EEG spectrum," Electroencephalography Clinical Neurophysiol., vol. 86, no. 1, pp. 23-35, 1993.

[7] W. Klimesch, "EEG alpha and theta oscillations reflect cognitive and memory performance: A review and analysis," Brain Res. Rev., vol. 29, pp. 169-195, 1999

[8] M. A. Schier, "Changes in EEG alpha power during simulated driving “ ,2000.

[9] Jim Horne and Louise Reyner, Sleep Related Vehicle Accidents, Sleep Research Laboratory, Loughborough University, 2000.

[10] G.E. Birch and S.G. Mason. Brain-Computer Interface Research at the Neil Squire Foundation, IEEE Trans. Rehab. Eng., 8(2), 193$95,2000$.

[11] H. J. Eoh, M. K. Chung, and S. H. Kim, "Electroencephalographic study of drowsiness in simulated driving with sleep deprivation," Int.J. Ind. Ergonom., vol. 35, pp. 307-320, 2005.

[12] Vila Bryan "Sleep Deprivation“ NIJ Journal no.262,Mar 27, 2009.

[13] "Sleep Facts and Stats"National sleep foundation.http://www.sleepfoundation.org .

[14] M. Matousek and I. Petersen, "A method for assessing alertness fluctuations from EEG spectra," Electroencephalography Clin. Neurophysiol., vol. 55, no. 1, pp. 108-113, 1983.

[15] S. Roberts, I. Rezek, R. Everson, H. Stone, S. Wilson, and C. Alford,“Automated assessment of vigilance using committees of radial basis function analysers,” Proc. Inst. Elect. Eng., Sci., Meas. Technol., vol.147, no. 6, pp. 333-338, 2000.

[16] H. J. Eoh, M. K. Chung, and S. H. Kim, "Electroencephalographic study of drowsiness in simulated driving with sleep deprivation," Int. J. Ind. Ergonom., vol. 35, pp. 307-320, 2005. 